\title{
An Instability Phenomenon in Hele-Shaw Displacements
}

\author{
Gelu Pasa ${ }^{1}$ \\ 1 "Simion Stoilow" Institute of Mathematics of Romanian Academy, Bucharest, Romania \\ Correspondence: Gelu Pasa, "Simion Stoilow" Institute of Mathematics of Romanian Academy, Calea Grivitei 21, \\ Bucuresti S1, Romania, Tel: 04-021-319-6504. E-mail: pasa.gelu@imar.ro
}

Received: August 30, 2017 Accepted: September 14, 2017 Online Published: October 9, 2017

doi:10.5539/jmr.v9n6p14

URL: https://doi.org/10.5539/jmr.v9n6p14

\begin{abstract}
We study the linear instability of the displacement of an Oldroyd-B fluid by air in a Hele-Shaw cell, motivated by possible applications in Secondary Oil Recovery (SOR) process. Most numerical methods have usually failed when the Weissenberg numbers $W_{i}$ (appearing in the constitutive relations) are near 1. We get an approximate formula of the growth rate with a blow-up for some particular $W_{i}=O(1)$. Therefore the instability at large Weissenberg numbers is due to the model, and not to the computational methods. Our growth rate is quite similar to Saffman-Taylor's formula (obtained when a Newtonian liquid is displaced by air) if $W_{1}=W_{2}$.
\end{abstract}

Keywords: Oldroyd-B fluids, Hele-Shaw displacement, linear stability

\section{Introduction}

A Hele-Shaw cell is a technical device consisting of two parallel plates, located at a small distance from each other. With some usual hypothesis - see (Hele-Shaw, 1898) - the averaged (across the plates) velocities of a Stokes flow are verifying an equation quite similar with the Darcy law for the flow in a porous medium, whose permeability is depending on the distance between the plates - see (Bear, 1972; Bird \& Stewart, 1960; Lamb, 1933).

We can consider two immiscible fluids with different viscosities in a Hele-Shaw cell. The "contact" zone is a small region, where the viscosity display a (continuous) large variation. In the Hele-Shaw model, this zone is replaced by a sharp interface, where a viscosity jump exists. This model can be used for study the displacement process similar with the Secondary Oil Recovery (SOR) procedure: the oil (at low pressure) from a porous medium is pushed by a second fluid. The linear stability of the interface between two immiscible fluids of Darcy type in a Hele-Shaw cell or porous medium was studied in (Saffman \& Taylor, 1958). The obtained growth constant is giving us the well-known SaffmanTaylor instability criterion: the interface is unstable if the displacing fluid is less viscous. The usual displacing fluids (some polymer-solute) used in (SOR) and the oil in a porous reservoir are often non-Newtonian fluids - the constitutive relations between the stress and the strain-rate tensors are non linear. Then seems be useful to study the "Saffman-Taylor" instability in the case of such fluids.

A large number of results in the field of non-Newtonian fluids are given in (Guillope \& Saut, 1992; Nittman et all, 1985; Renardy, 2000; Schowlater, 1978; Truesdell \& Noll, 1965; Zhao \& Maher, 1993). The displacement of Maxwell upperconvected fluids by air in a Hele-Shaw cell are studied numerically in (Mora \& Manna, 2009; Mora \& Manna, 2010).

Some numerical results concerning the instability of the interface between air (as a displacing fluid) and an Oldroyd-B fluid in a Hele-Shaw were obtained in (Wilson, 1990). Here a blow-up of the growth constant for $W_{2}=0, W_{1}>2.5$ was reported, similar with the fractures observed in the flows of some complex fluids in Hele-Shaw cells - see (Nase et all, 2008), (Nittman et all, 1985; Zhao \& Maher, 1993).

In this paper we study the modal linear stability of the interface appearing when an Oldroyd-B fluid is displaced by air in a Hele-Shaw cell. The Weissenberg numbers $W_{i}$ appear in the constitutive relations (3) and we consider $W_{i}=O(1)$. The basic flow (the same as in (Wilson, 1990)) is described in section 2. The perturbations system is derived in section 3. We use the particular perturbations (26), depending on the arbitrary parameter $\alpha$. If $\alpha$ checks the condition (43), then we can neglect some terms in the constitutive relations and we get approximate expressions of some components of the extra-stress tensor. An approximate formula of the growth rate of perturbations is given in section 4. We get dispersion curves which are similar with numerical results given in (Wilson, 1990). Our growth constant is similar with the SaffmanTaylor's formula if $W_{1}=W_{2}$. The main point is following: the particular perturbations (26) lead us to the blow-up of the growth constant (48) for $W_{1}-W_{2} \approx 0.4$. Then the large Weissenberg number instability is due to the model, and not to the computational methods. We conclude in section 5, where we further deomostrate that our basic flow becomes Newtonian if $W_{1}=W_{2}$ (that means the relaxation and retardation time constants are equal in the constitutive relations (3)). 


\section{The Oldroyd-B Fluid and the Basic Flow}

We consider a horizontal Hele-Shaw cell, parallel with the $x O y$ plane and neglect the gravity. An Oldroyd-B fluid is displaced by air in the positive direction of $O x$. The distance between plates is denoted by $b$ and the cell length cell is $l$ such that $\epsilon=b / l<<1$. We use the following notations:

the extra-stress and strain-rate tensors: $\underline{\tau}, \underline{\mathbf{S}}$;

the velocity, pressure and the fluid viscosity: $\underline{\mathbf{u}}=(\underline{u}, \underline{w}, \underline{w}), \underline{p}, \mu$;

the relaxation and retardation (time) constants: $\lambda_{1}, \lambda_{2}$;

the matrix containing the velocity derivatives: $\underline{\mathbf{V}}$;

the strain-rate tensor: $\underline{\mathbf{S}}:=\left(\underline{\mathbf{V}}+\underline{\mathbf{V}}^{T}\right) / 2 ; \quad\left(\underline{\mathbf{V}}_{i j}\right)^{T}:=\underline{\mathbf{V}}_{j i}$.

The flow equations, the free-divergence condition and the constitutive relations are

$$
\begin{gathered}
\underline{p}_{x}=\underline{\tau}_{11, x}+\underline{\tau}_{12, y}+\underline{\tau}_{13, z}, \quad \underline{p}_{y}=\underline{\tau}_{21, x}+\underline{\tau}_{22, y}+\underline{\tau}_{23, z}, \quad \underline{p}_{z}=\underline{\tau}_{31, x}+\underline{\tau}_{32, y}+\underline{\tau}_{33, z}, \\
\underline{u}_{x}+\underline{v}_{y}+\underline{w}_{z}=0, \\
\underline{\tau}^{+}+\lambda_{1} \underline{\tau}^{\nabla}=2 \mu\left[\underline{\mathbf{S}}+\lambda_{2} \underline{\mathbf{S}}^{\nabla}\right], \quad \lambda_{1}>\lambda_{2} \geq 0, \\
\underline{\tau}^{\nabla}=\underline{\mathbf{u}} \cdot \nabla \underline{\tau}-\left(\underline{\mathbf{V} \tau}+\underline{\mathbf{V}}^{T}\right), \quad \underline{\mathbf{S}}^{\nabla}=\underline{\mathbf{u}} \cdot \nabla \underline{\mathbf{S}}-\left(\underline{\mathbf{V}}+\underline{\mathbf{S V}}^{T}\right) .
\end{gathered}
$$

The last two relations are not containing the partial time derivatives $\underline{\tau}_{t}$ and $\underline{\mathbf{S}}_{t}$, because we consider a steady flow. $\underline{p}_{x}, \underline{p}_{y}, \underline{p}_{z}$, $\underline{\tau}_{i j, x}, \underline{\tau}_{i j, y}, \underline{\tau}_{i j, z}$ are denoting the $x, y, z$ partial derivatives of $\underline{\tau}_{i j}$ and $\underline{p}$. The following boundary conditions are used:

a) No-slip condition for the velocity components on the plates.

b) Laplace's law in the neighborhood of the basic air-fluid interface.

We study the linear stability of the following basic flow, denoted by the super index ${ }^{0}$ :

$$
\begin{array}{cc}
\nabla p^{0}=\left(p_{x}^{0}(x), 0,0\right), & \mathbf{v}^{0}=\left(u^{0}(z), 0,0\right), \\
\mathbf{V}^{0}=\left(\begin{array}{ccc}
0 & 0 & u_{z}^{0} \\
0 & 0 & 0 \\
0 & 0 & 0
\end{array}\right), \quad \mathbf{S}^{0}=\frac{1}{2}\left(\mathbf{V}^{0}+\mathbf{V}^{0 T}\right) .
\end{array}
$$

The basic extra-stress tensor is given by the following equation

$$
\tau^{0}-\lambda_{1}\left(\mathbf{V}^{0} \tau^{0}+\tau^{0} \mathbf{V}^{0 T}\right)=\mu\left\{2 \mathbf{S}^{0}-\lambda_{2}\left(2 \mathbf{V}^{0} \mathbf{S}^{0}+2 \mathbf{S}^{0} \mathbf{V}^{0 T}\right)\right\},
$$

where

$$
\mathbf{V}^{0} \tau^{0}+\tau^{0} \mathbf{V}^{0 T}=\left(\begin{array}{ccc}
2 u_{z}^{0} \tau_{31}^{0} & u_{z}^{0} \tau_{32}^{0} & u_{z}^{0} \tau_{33}^{0} \\
u_{z}^{0} \tau_{32}^{0} & 0 & 0 \\
u_{z}^{0} \tau_{33}^{0} & 0 & 0
\end{array}\right), 2\left(\mathbf{V}^{0} \mathbf{S}^{0}+\mathbf{S}^{0} \mathbf{V}^{0 T}\right)=\left(\begin{array}{ccc}
2\left(u_{z}^{0}\right)^{2} & 0 & 0 \\
0 & 0 & 0 \\
0 & 0 & 0
\end{array}\right)
$$

Therefore the components of the basic extra-stress are

$$
\begin{gathered}
\tau_{33}^{0}=0, \quad \tau_{22}^{0}=0, \quad \tau_{23}^{0}=0, \quad \tau_{12}^{0}=0, \\
\tau_{13}^{0}=\mu u_{z}^{0}, \quad \tau_{11}^{0}=2\left(\lambda_{1}-\lambda_{2}\right) \mu\left(u_{z}^{0}\right)^{2}
\end{gathered}
$$

and we get the basic flow equations:

$$
p_{x}^{0}=\tau_{11, x}^{0}+\tau_{12, y}^{0}+\tau_{13, z}^{0}, \quad p_{y}^{0}=\tau_{21, x}^{0}+\tau_{22, y}^{0}+\tau_{23, z}^{0}, \quad p_{z}^{0}=\tau_{31, x}^{0}+\tau_{32, y}^{0}+\tau_{33, z}^{0} .
$$

The equations (5) - (10) (used also in (Wilson, 1990)) give us

$$
p_{z}^{0}=0, p_{y}^{0}=0, p_{x}^{0}(x)=\tau_{13, z}^{0}(z)
$$

We conclude that a negative constant $G$ exists such that $p_{x}^{0}(x)=\mu u_{z z}^{0}=G$, therefore the basic velocity $u^{0}$ can be obtained in terms of $G$ :

$$
u^{0}=\frac{1}{2 \mu} p_{x}^{0}\left(z^{2}-b z\right)=\frac{1}{2 \mu} G\left(z^{2}-b z\right) .
$$


The following characteristic velocity $U$ is introduced

$$
U=<u^{0}>:=\frac{1}{b} \int_{0}^{b} u^{0} d z=-\frac{b^{2}}{12 \mu} p_{x}^{0},
$$

then we have the relations

$$
u^{0}=(G / 2 \mu) z(z-b)=-\left(6 U / b^{2}\right) z(z-b)
$$

The basic air-fluid interface is

$$
x=<u^{0}>t=U t .
$$

As in (Wilson, 1990), the basic pressure can depend on the time $t$ :

$$
p^{0}=G\left(x-<u^{0}>t\right)=G(x-U t), \quad x><u^{0}>t=U t .
$$

In the following we consider the moving coordinate system $\bar{x}=x-U t$, then the basic interface is $\bar{x}=0$. But (with no confusion) we still use the notation $x=0$ for the basic interface.

\section{The Perturbations System}

The small perturbations of the basic solution are denoted by $u, v, w, p, \tau$,

$$
\mathbf{V}=\left(\begin{array}{ccc}
u_{x} & u_{y} & u_{z} \\
v_{x} & v_{y} & v_{z} \\
0 & 0 & 0
\end{array}\right), \quad \mathbf{S}=\left(\mathbf{V}+\mathbf{V}^{T}\right) / 2
$$

The perturbation of the basic interface is denoted by $\eta$. We consider that a fluid element that was originally on the interface remains here, then it follows

$$
\eta_{t}=u
$$

(in other words, the interface is material).

In the frame of the linear stability, the free-divergence relation is also verified by the components of the velocity perturbation, then $u_{x}+v_{y}+w_{z}=0$. We integrate across the plates, we use the condition $w=0$ on $z=0, z=b$, then we get

$$
\int_{0}^{b}\left(u_{x}+v_{y}\right)=0
$$

In this paper we consider the particular perturbations such that

$$
u_{x}+v_{y}=0,
$$

(which verify the above condition) and we get $w_{z}=0$. Then the boundary conditions are giving us $w=0$, which is obtained in (Wilson, 1990) by using a numerical method .

We introduce the small perturbations in the constitutive equations and in the expressions of the upper convected derivatives and get

$$
\begin{gathered}
\tau^{0}+\tau+\lambda_{1}\left(\tau^{0}+\tau\right)^{\nabla}=2 \mu\left[\mathbf{S}^{0}+\mathbf{S}+\lambda_{2}\left(\mathbf{S}^{0}+\mathbf{S}\right)^{\nabla}\right], \\
\left(\tau^{0}+\tau\right)^{\nabla}=u^{0} \tau_{x}-\left[\mathbf{V}^{0} \tau^{0}+\tau^{0} \mathbf{V}^{0 T}\right]-\left[\mathbf{V}^{0} \tau+\mathbf{V} \tau^{0}+\tau^{0} \mathbf{V}^{T}+\tau \mathbf{V}^{0 T}\right] \\
\left(\mathbf{S}^{0}+\mathbf{S}\right)^{\nabla}=u^{0} \mathbf{S}_{x}-\left[\mathbf{V}^{0} \mathbf{S}^{0}+\mathbf{S}^{0} \mathbf{V}^{0 T}\right]-\left[\mathbf{V}^{0} \mathbf{S}+\mathbf{V} \mathbf{S}^{0}+\mathbf{S}^{0} \mathbf{V}^{T}+\mathbf{S} \mathbf{V}^{0 T}\right]
\end{gathered}
$$

In the frame of the linear stability (that means by neglecting the second order terms in perturbations) it follows

$$
\begin{gathered}
\tau+\lambda_{1}\left(u^{0} \tau_{x}-\mathbf{E}\right)=\mu\left\{2 \mathbf{S}+\lambda_{2}\left(u^{0} 2 \mathbf{S}_{x}-\mathbf{F}\right)\right\}, \\
\mathbf{E}:=\mathbf{V}^{0} \tau+\mathbf{V} \tau^{0}+\tau^{0} \mathbf{V}^{T}+\tau \mathbf{V}^{0 T}, \\
\mathbf{F}:=2\left[\mathbf{V}^{0} \mathbf{S}+\left(\mathbf{V}^{0} \mathbf{S}\right)^{T}+\mathbf{V} \mathbf{S}^{0}+\left(\mathbf{V} \mathbf{S}^{0}\right)^{T}\right] .
\end{gathered}
$$

We have

$$
\tau^{0} \mathbf{V}^{T}=\left(\mathbf{V} \tau^{0}\right)^{T}, \quad \tau \mathbf{V}^{0 T}=\left(\mathbf{V}^{0} \tau\right)^{T}, \quad \mathbf{S}^{0} \mathbf{V}^{T}=\left(\mathbf{V} \mathbf{S}^{0}\right)^{T}, \quad \mathbf{S} \mathbf{V}^{0 T}=\left(\mathbf{V}^{0} \mathbf{S}\right)^{T}
$$


and by using (6), (9) and (17) we get

$$
\begin{gathered}
\mathbf{V}^{0} \tau=\left(\begin{array}{ccc}
u_{z}^{0} \tau_{31} & u_{z}^{0} \tau_{32} & u_{z}^{0} \tau_{33} \\
0 & 0 & 0 \\
0 & 0 & 0
\end{array}\right) ; \quad \mathbf{V} \tau^{0}=\left(\begin{array}{ccc}
u_{x} \tau_{11}^{0}+u_{z} \tau_{31}^{0} & 0 & u_{x} \tau_{13}^{0} \\
v_{x} \tau_{11}^{0}+v_{z} \tau_{31}^{0} & 0 & v_{x} \tau_{13}^{0} \\
0 & 0 & 0
\end{array}\right) ; \\
\mathbf{E}=\left(\begin{array}{cccc}
\mathbf{V}^{0} \mathbf{S}=\left(\begin{array}{ccc}
u_{z}^{0} u_{z} & u_{z}^{0} v_{z} & 0 \\
0 & 0 & 0 \\
0 & 0 & 0
\end{array}\right) ; \quad 2 \mathbf{V} \mathbf{S}^{0}=\left(\begin{array}{ccc}
u_{z} u_{z}^{0} & 0 & u_{x} u_{z}^{0} \\
v_{z} u_{z}^{0} & 0 & v_{x} u_{z}^{0} \\
0 & 0 & 0
\end{array}\right) ; \\
\left(u_{z}^{0} \tau_{31}+u_{32} \tau_{11}^{0}+v_{13}^{0} u_{z}\right) & \left(u_{z}^{0} \tau_{32}+v_{x} \tau_{11}^{0}+v_{z} \tau_{13}^{0}\right) & \left(u_{z}^{0} \tau_{33}+u_{x} \tau_{13}^{0}\right) \\
\left(u_{z}^{0} \tau_{33}+u_{x} \tau_{13}^{0}\right) & 0
\end{array}\right) \\
\mathbf{F}=\left(\begin{array}{ccc}
4 u_{z}^{0} u_{z} & 2 u_{z}^{0} v_{z} & u_{x} u_{z}^{0} \\
2 u_{z}^{0} v_{z} & 0 & v_{x} u_{z}^{0} \\
u_{x} u_{z}^{0} & v_{x} u_{z}^{0} & 0
\end{array}\right) .
\end{gathered}
$$

\section{The Sability Analysis}

We consider the following perturbations of the basic solution:

$$
\begin{gathered}
u=f(z) E X P \cos (n y), \quad v=f(z) E X P \sin (n y), \quad E X P=\exp (-n[\alpha+x]+\sigma t), \\
\sigma=\text { growth constant }, \quad f(z)=\beta u^{0}(z), \quad \beta=O\left(\epsilon^{2}\right), \quad \text { dimension of } \alpha=\text { length. }
\end{gathered}
$$

We obtain a formula for the growth constant $\sigma$ corresponding to the perturbations (26), which is displaying a blow-up (in terms of the wavenumbers $n$ ) for some particular values of the Weissenberg numbers.

Near the basic interface $x=0$ we use the following Laplace's law as in (Renardy, 2000; Wilson, 1990) (see (13) and (18) for definitions of $G, \eta)$ :

$$
(G<\eta>+<p>)-<\tau_{11}>=\gamma\left(<\eta_{y y}+\eta_{z z}>\right), \quad \eta=u / \sigma .
$$

Here $\left(\eta_{y y}+\eta_{z z}\right)$ is the approximate expression of the total curvature of the perturbed interface and $\gamma$ is the surface tension on the interface. The above relation gives the growth constant expression:

$$
\sigma=\frac{\gamma<u_{y y}+u_{z z}>-G<u>}{<p-\tau_{11}>} .
$$

The problem is to compute $<p-\tau_{11}>$ in terms of the basic and perturbed velocities. For this, in the following we search a particular solution $\tau_{33}, \tau_{13}, \tau_{23}, \tau_{12}, p_{x}, p_{z}, \tau_{11}$ in terms of $u, v, u^{0}, v^{0}$.

We define the dimensionless quantities

$$
\begin{gathered}
x^{\prime}=x / l, \quad y^{\prime}=y / l, \quad z^{\prime}=z / b, \quad \epsilon=b / l<<1, \quad u^{\prime}=u / U, \quad v^{\prime}=v / U, \\
p^{\prime}=p(l / \mu U), \quad \gamma^{\prime}=\gamma(1 / \mu U), \quad n^{\prime}=n l, \quad \sigma^{\prime}=\sigma(l / U), \quad \alpha^{\prime}=\alpha / l, \quad t^{\prime}=t(U / l), \\
\left\{\tau_{11}^{\prime}, \tau_{12}^{\prime}, \tau_{22}^{\prime}\right\}=\left\{\tau_{11}, \tau_{12}, \tau_{22}\right\}(l / \mu U), \quad\left\{\tau_{13}^{\prime}, \tau_{23}^{\prime}, \tau_{33}^{\prime}\right\}=\left\{\tau_{13}, \tau_{23}, \tau_{33}\right\}(b / \mu U), \\
W_{i}=\lambda_{i}(U / b),
\end{gathered}
$$

where $W_{i}$ are the Weissenberg numbers. From (12), (13) and (26) we get

$$
\begin{gathered}
u^{\prime}=\beta u^{0^{\prime}} E X P^{\prime} \cos \left(n^{\prime} y^{\prime}\right)=\beta 6 z^{\prime}\left(1-z^{\prime}\right) E X P^{\prime} \cos \left(n^{\prime} y^{\prime}\right), \\
v^{\prime}=\beta u^{0^{\prime}} E X P^{\prime} \cos \left(n^{\prime} y^{\prime}\right)=\beta 6 z^{\prime}\left(1-z^{\prime}\right) E X P^{\prime} \sin \left(n^{\prime} y^{\prime}\right), \\
E X P^{\prime}=\exp \left(-n^{\prime}\left[\alpha^{\prime}+x^{\prime}\right]+\sigma^{\prime} t^{\prime}\right) .
\end{gathered}
$$

In the following we use only dimensionless quantities, then we omit the'.

The flow equations and the dimensionless quantities (28) give us

$$
p_{x} \frac{\mu U}{l^{2}}-\tau_{11, x} \frac{\mu U}{l^{2}}=\tau_{12, y} \frac{\mu U}{l^{2}}+\tau_{13, z} \frac{\mu U}{b^{2}}, \quad p_{x}-\tau_{11, x}=\tau_{12, y}+\tau_{13, z} \frac{1}{\epsilon^{2}},
$$




$$
p-\tau_{11}=(-1 / n)\left\{\tau_{12, y}+\tau_{13, z} \frac{1}{\epsilon^{2}}\right\}
$$

Proposition 1. We prove that

$$
\tau_{33}=0, \quad \tau_{31}=u_{z}, \quad \tau_{32}=v_{z}
$$

are possible solutions of the perturbations system (22).

Proof. From (22) and (28) we get

$$
\begin{gathered}
\tau_{33}+W_{1} \epsilon u^{0} \tau_{33, x}=0, \\
\tau_{31}+W_{1} \epsilon u^{0} \tau_{31, x}-W_{1} \epsilon\left(u_{z}^{0} \tau_{33}+u_{x} \tau_{13}^{0}\right)=u_{z}+W_{2} \epsilon\left(u^{0} u_{z x}-u_{x} u_{z}^{0}\right), \\
\tau_{32}+W_{1} \epsilon\left(u^{0} \tau_{32, x}-\tau_{13}^{0} v_{x}\right)=v_{z}+W_{2} \epsilon\left(u^{0} v_{z x}-v_{x} u_{z}^{0}\right) .
\end{gathered}
$$

The equation (32) gives us the possible solution $\tau_{33}=0$. We use (26), then in the right part of (33) - (34) we get $W_{2} \epsilon\left(u^{0} u_{z x}-u_{z}^{0} u_{x}\right)=0, W_{2} \epsilon\left(u^{0} v_{z x}-v_{x} u_{z}^{0}\right)=0$. If $\tau_{31}=u_{z}, \tau_{32}=v_{z}$, in the left part of (33), (34) we get

$$
\begin{gathered}
W_{1} \epsilon\left(u^{0} \tau_{31, x}+u_{z}^{0} \tau_{33}-u_{x} \tau_{13}^{0}\right)=W_{1} \epsilon\left(u^{0} u_{z x}-u_{x} u_{z}^{0}\right)=0 \\
W_{1} \epsilon\left(u^{0} \tau_{32, x}-\tau_{13}^{0} v_{x}\right)=W_{1} \epsilon\left(u^{0} v_{z x}-v_{x} u_{z}^{0}\right)=0 .
\end{gathered}
$$

Then the system (32) - (34) is verified by the expressions (31). As a consequence, we obtain

$$
p_{z}=\tau_{31, x}+\tau_{32, y}=\mu\left(u_{z x}+v_{z y}\right)=0
$$

From $(31)_{2}$ we get $\tau_{31, z}=u_{z z}$. We use (13), (27), (30) and we obtain the dimensionless from of the Laplace's law as follows:

$$
\begin{gathered}
\frac{-12 \mu U}{b^{2}} \cdot \frac{<U u>}{\sigma(U / l)}+\frac{\mu U}{l}<p-\tau_{11}>=\frac{\gamma \mu U}{\sigma(U / l)}\left[<U u_{y y}>\frac{1}{l^{2}}+<U u_{z z}>\frac{1}{b^{2}}\right], \\
\frac{-12<u>}{\epsilon^{2}} \frac{<}{\sigma}+(-1 / n)\left\{\tau_{12, y}+u_{z z} \frac{1}{\epsilon^{2}}\right\}=\frac{\gamma}{\sigma}<u_{y y}+u_{z z} \frac{1}{\epsilon^{2}}>.
\end{gathered}
$$

The term $<u_{y y}>$ can not be neglected, being related with the curvature of the perturbed interface.

Proposition 2. If $\exp (\sigma t)<1$, then $\forall x \geq 0$ we have

$$
\begin{array}{ll}
\max _{n}\left\{v_{x}\right\}=\frac{3 \beta}{2 \alpha e}, & \max _{n}\left\{v_{x x}\right\}=\frac{6 \beta}{\alpha^{2} e^{2}}, \\
\max _{n}\left\{u_{x}\right\}=\frac{3 \beta}{2 \alpha e}, & \max _{n}\left\{v_{x x x}\right\}=\frac{81 \beta}{2 \alpha^{3} e^{3}}, \\
\max _{n}\left\{u_{x x}\right\}=\frac{6 \beta}{\alpha^{2} e^{2}}, & \max _{n}\left\{u_{x x x}\right\}=\frac{81 \beta}{2 \alpha^{3} e^{3}} .
\end{array}
$$

Proof. From (14) we have $u^{0}=6 z(1-z) \leq 6 \cdot(1 / 4)=3 / 2$ and get

$$
u_{x} \leq(3 \beta / 2) n \exp (-n \alpha), \quad u_{x x} \leq(3 \beta / 2) n^{2} \exp (-n \alpha), \quad u_{x x x} \leq(3 \beta / 2) n^{3} \exp (-n \alpha) .
$$

Consider the function $F 1(n)=n e^{-n \alpha}$, then

$$
d F 1 / d n=(1-n \alpha) e^{-n \alpha}, \quad d F 1 / d n(1 / \alpha)=0 \Rightarrow \max _{n} F 1=F 1(1 / \alpha)=1 /(\alpha e) .
$$

The maximal values of $n^{2} e^{-n \alpha}, n^{3} e^{-n \alpha}$ give us the relations (37). As a consequence, when $\alpha>81 /(12 e)$ we obtain $\max _{n}\left\{u_{x x x}\right\}<\max _{n}\left\{u_{x x}\right\}$.

Remark 1. In the Figure 1 we see that $\epsilon n^{3} \exp (-\alpha n)$ can be neglected in front $n^{2} \exp (-\alpha n)$, when $\alpha=2, \epsilon=0.02$. In practice, we have $\epsilon \approx 10^{-3}$ and numerical estimates give $\epsilon n^{3} \exp (-\alpha n)<0.0003$. This is the point in our procedure for obtaining an approximate growth constant.

Proposition 3. If $\exp (\sigma t)<1, \beta=O\left(\epsilon^{2}\right),\left(W_{1}-W_{2}\right)=O(1), \alpha>11\left(W_{2}-W_{1}\right) / e$, then $\tau_{12, y}$ can be approximated by the formula

$$
\tau_{12, y}=\left(u_{y}+v_{x}\right)_{y}+2\left(W_{1}-W_{2}\right) u_{z}^{0} v_{z y} / \epsilon
$$

Proof. The constitutive relations (22) and the dimensionless quantities (28) give us

$$
\tau_{12, y} \frac{\mu U}{l^{2}}+\lambda_{1} u^{0} \tau_{12, x y} \frac{\mu U^{2}}{l^{3}}-\lambda_{1}\left[u_{z}^{0} \mu v_{z y} \frac{U^{2}}{b^{2} l}+v_{x y} 2 \mu\left(\lambda_{1}-\lambda_{2}\right)\left(u_{z}^{0}\right)^{2} \frac{U^{3}}{l^{2} b^{2}}+\mu u_{z}^{0} v_{z y} \frac{U^{2}}{b^{2} l}\right]=
$$




$$
\mu\left\{\left(u_{y}+v_{x}\right)_{y} \frac{U}{l^{2}}+\lambda_{2} u^{0}\left(u_{y}+v_{x}\right)_{x y} \frac{U^{2}}{l^{3}}-2 \lambda_{2} u_{z}^{0} v_{z y} \frac{U^{2}}{b^{2} l}\right\} .
$$

Recall $W_{i}=\lambda_{i} U / b, \quad \epsilon=b / l$, then we get

$$
\begin{gathered}
\tau_{12, y}+W_{1} u^{0} \tau_{12, x y} \epsilon-W_{1}\left[2 u_{z}^{0} v_{z y} / \epsilon+2\left(W_{1}-W_{2}\right) v_{z y}\left(u_{z}^{0}\right)^{2}\right]= \\
\left(u_{y}+v_{x}\right)_{y}+W_{2} u^{0}\left(u_{y}+v_{x}\right)_{x y} \epsilon-2 W_{2} u_{z}^{0} v_{z y} / \epsilon .
\end{gathered}
$$

We introduce the expression (38) in (39) and obtain

$$
\begin{gathered}
\tau_{12, y}=-W_{1} u^{0} \epsilon\left\{\left(u_{y}+v_{x}\right)_{y x}+2\left(W_{1}-W_{2}\right) u_{z}^{0} v_{z y x} / \epsilon\right\}+ \\
\left(u_{y}+x_{x}\right)_{y}+2\left(W_{1}-W_{2}\right) u_{z}^{0} v_{z y} / \epsilon+2 W_{1}\left(W_{1}-W_{2}\right) v_{x y}\left(u_{z}^{0}\right)^{2}+W_{2} u^{0}\left(u_{y}+v_{x}\right)_{x y} \epsilon .
\end{gathered}
$$

From the Fourier expansion (26) we have $2 W_{1}\left(W_{1}-W_{2}\right)\left[v_{x y}\left(u_{z}^{0}\right)^{2}-u^{0} u_{z}^{0} v_{z y x}\right]=0$, then the last relation (40) becomes

$$
\tau_{12, y}=\left(u_{y}+v_{x}\right)_{y}+2\left(W_{1}-W_{2}\right) u_{z}^{0} v_{z y} / \epsilon+\left(W_{2}-W_{1}\right) u^{0}\left(u_{y}+v_{x}\right)_{x y} \epsilon .
$$

The equation (14) and the dimensionless quantities (28) give us $u^{0}=6\left(z-z^{2}\right) \leq 3 / 2, \quad u_{z}^{0}=6(1-2 z) \leq 6$. We need estimates of the maximal values of the terms in the right part of the expression (41). We see that:

$$
u_{y y}, v_{x y} \text { contain the factor } n^{2} e^{-n \alpha}, \quad u_{y x y}, v_{x x y} \text { contain the factor } n^{3} e^{-n \alpha} .
$$

Then we can use Proposition 2 and get

$$
\begin{gathered}
\max _{n}\left(u_{y}+v_{x}\right)_{y}=2 \cdot \frac{3 \beta}{2} \cdot \frac{4}{\alpha^{2} e^{2}}=\frac{12 \beta}{\alpha^{2} e^{2}}=\frac{12}{\alpha^{2} e^{2}} \epsilon^{2} \\
\max _{n}\left(W_{1}-W_{2}\right) u_{z}^{0} v_{z y} \frac{1}{\epsilon}=\left(W_{1}-W_{2}\right) \frac{36 \beta}{\alpha e \epsilon}=O(1) \frac{36}{\alpha e} \epsilon \\
\max _{n}\left(W_{2}-W_{1}\right) u^{0}\left(u_{y}+v_{x}\right)_{x y} \epsilon=\left|W_{2}-W_{1}\right| \frac{9 \beta}{2} \cdot \frac{27}{\alpha^{3} e^{3}} \epsilon=O(1) \frac{243}{2 \alpha^{3} e^{3}} \epsilon^{3} .
\end{gathered}
$$

The condition

$$
\alpha>11\left|W_{2}-W_{1}\right| / e
$$

gives us

$$
\alpha>\frac{243\left|W_{2}-W_{1}\right|}{24 e} \text { and } \frac{243 \beta\left|W_{2}-W_{1}\right|}{2 \alpha^{3} e^{3}} \epsilon<\frac{12 \beta}{\alpha^{2} e^{2}} \epsilon .
$$

As $\epsilon<<1$, from (42) - (44) we get

$$
\max _{n}\left(W_{2}-W_{1}\right) u^{0}\left(u_{y}+v_{x}\right)_{x y} \epsilon<\max _{n}\left(u_{y}+v_{x}\right)_{y} \epsilon<<\max _{n}\left(u_{y}+v_{x}\right)_{y} .
$$

We conclude that $\left(W_{2}-W_{1}\right) u^{0}\left(u_{y}+v_{x}\right)_{x y} \epsilon$ can be neglected in front of $\left(u_{y}+v_{x}\right)_{y}$ and (41) gives the relation (38).

Remark 2. The above result is an important improvement compared with (Wilson, 1990), where instead of (26) was used the expansion

$$
u \propto \exp (-n x+\sigma t \cos (n y), \quad v \propto \exp (-n x+\sigma t) \sin (n y) .
$$

By using (45), the second and third partial derivatives of $(u, v)$ with respect to $x, y$ contain the factors $n^{2} \exp (-n x+\sigma t)$ and $n^{3} \exp (-n x+\sigma t)$, which are not bounded in terms of $n$ when $x \rightarrow 0$, even if $\exp (\sigma t)<1$. Moreover, the expansion (45) is not giving the explicit expression of the perturbations amplitude, which in our paper is $6 \beta|z(1-z)|$. Therefore the expansion (26) and the condition (43) allow us to avoid the singularity near $x=0$ and to neglect, in a rigorous way, some terms in the constitutive relations.

The expression (38) of $\tau_{12, y}$ allow us to get the growth constant formula, as follows. The relations (29), (38) give us

$$
\begin{gathered}
p-\tau=(-1 / n)\left\{\left(u_{y}+v_{x}\right)_{y}+2\left(W_{1}-W_{2}\right) u_{z}^{0} v_{z y} \frac{1}{\epsilon}+u_{z z} \frac{1}{\epsilon^{2}}\right\}= \\
(-1 / n)\left[O\left(\epsilon^{2}\right)+O(\epsilon)+O(1)\right] .
\end{gathered}
$$

Consider $O(\gamma)=1$. Then (36) and (46) give the magnitudes of the terms appearing in the dimensionless Laplace's law :

$$
\frac{O(1)}{\sigma}-\frac{1}{n}\left\{O\left(\epsilon^{2}\right)+O(\epsilon)+O(1)\right\}=\frac{O(1)}{\sigma}\left\{O\left(\epsilon^{2}\right)+O(1)\right\} .
$$


We insert (46) in (36). As $<u^{0}>=1,<\left(u_{z}^{0}\right)^{2}>=12$, we get

$$
\frac{-12}{\epsilon^{2} \sigma}+(-1 / n)\left\{-2 n^{2}+24 n\left(W_{1}-W_{2}\right) \frac{1}{\epsilon}-12 \frac{1}{\epsilon^{2}}\right\}=\frac{\gamma}{\sigma}\left(-n^{2}-12 \frac{1}{\epsilon^{2}}\right)
$$

From the last relation we obtain the following

Proposition 4. The growth rate $\sigma$ corresponding to the approximate solution (38) is given by the expression

$$
\sigma=\frac{n(1-\gamma)-\gamma\left(\epsilon^{2} / 12\right) n^{3}}{n^{2}\left(\epsilon^{2} / 6\right)-2 n\left(W_{1}-W_{2}\right) \epsilon+1} .
$$

Remark 3. In the case $\exp (\sigma t)<1$, from (48) we obtain the following important results:

a) The denominator of (48) is $\neq 0$ for $W_{1}-W_{2}<1 / \sqrt{6} \approx 0.408$. Indeed, we have

$$
W_{1}-W_{2}<1 / \sqrt{6} \Rightarrow \Delta=\left(W_{1}-W_{2}\right) \epsilon^{2}-\left(\epsilon^{2} / 6\right)<0 .
$$

b) $\sigma \rightarrow \infty$ when $W_{2}=0, \quad W_{1}<1 / \sqrt{6}, \quad W_{1} \rightarrow 1 / \sqrt{6}$.

c) If $0.3<W 1<0.408$ and $W_{2}=0$ we have a destabilization effect compared with the Saffman-Taylor growth constant $\sigma_{S-T}$ below:

$$
\sigma_{S-T}=n-\gamma\left(\epsilon^{2} / 12\right) n^{3}
$$

In Figure 2 we plot $\sigma$ given by (48) (on the vertical axis) in terms of $n$ (on the horizontal axis), for $\gamma=0.1, \epsilon=0.006$, $W_{2}=0$ and $W_{1}$ increasing from 0.1 until 0.38. The plots are similar with the numerical results given in (Wilson, 1990). We also also plotted the Saffman-Taylor growth rate (49).

Proposition 5. If the hypothesis of Proposition 3 hold, then $\tau_{11, x}$ can be approximated by the formula

$$
\tau_{11, x}=2 u_{x x}+4\left(W_{1}-W_{2}\right) u_{z}^{0} u_{z x} / \epsilon .
$$

Proof. The dimensionless form of the constitutive relations (22) is

$$
\begin{gathered}
\tau_{11, x} \frac{\mu U}{l^{2}}+\lambda_{1} u^{0} \tau_{11, x x} \frac{\mu U^{2}}{l^{3}}-2 \lambda_{1}\left[u_{z}^{0} \mu u_{z x} \frac{U^{2}}{b^{2} l}+u_{x x} 2 \mu\left(\lambda_{1}-\lambda_{2}\right)\left(u_{z}^{0}\right)^{2} \frac{U^{3}}{l^{2} b^{2}}+\mu u_{z}^{0} u_{z x} \frac{U^{2}}{b^{2} l}\right]= \\
\mu\left\{2 u_{x x} \frac{U}{l^{2}}+\lambda_{2} u^{0} 2 u_{x x x} \frac{U^{2}}{l^{3}}-4 \lambda_{2} u_{z}^{0} u_{z x} \frac{U^{2}}{b^{2} l}\right\},
\end{gathered}
$$

then we obtain

$$
\begin{gathered}
\tau_{11, x}+W_{1} u^{0} \tau_{11, x x} \epsilon-2 W_{1}\left[u_{z}^{0} u_{z x} / \epsilon+u_{x x} 2\left(W_{1}-W_{2}\right)\left(u_{z}^{0}\right)^{2}+u_{z}^{0} u_{z x} / \epsilon\right]= \\
2 u_{x x}+2 W_{2} u^{0} u_{x x x} \epsilon-4 W_{2} u_{z}^{0} u_{z x} / \epsilon .
\end{gathered}
$$

We insert the expression (50) in the last relation and get

$$
\begin{gathered}
\tau_{11, x}+W_{1} \epsilon u^{0}\left[2 u_{x x x}+4\left(W_{1}-W_{2}\right) u_{z}^{0} u_{z x x} / \epsilon\right]-2 W_{1}\left[u_{z}^{0} u_{z x} / \epsilon+u_{x x} 2\left(W_{1}-W_{2}\right)\left(u_{z}^{0}\right)^{2}+u_{z}^{0} u_{z x} / \epsilon\right]= \\
2 u_{x x}+2 W_{2} u^{0} u_{x x x} \epsilon-4 W_{2} u_{z}^{0} u_{z x} / \epsilon .
\end{gathered}
$$

As $f(z)=\beta u^{0}$, from (26) we have $u^{0} u_{z}^{0} u_{z x x}-u_{x x}\left(u_{z}^{0}\right)^{2}=0$, then it follows

$$
\tau_{11, x}=2 u_{x x}+4\left(W_{1}-W_{2}\right) u_{z}^{0} u_{z x} / \epsilon+2\left(W_{2}-W_{1}\right) \epsilon u^{0} u_{x x x} .
$$

As in Proposition 3, the condition (43) allows us to neglect $\left\{2\left(W_{2}-W_{1}\right) \epsilon u^{0} u_{x x x}\right\}$ in front of $\left\{2 u_{x x}\right\}$. Then the last above expresion of $\tau_{11, x}$ gives us the equation (50).

We compute now the expression of $p_{x}$, by using (38) and (50) :

$$
\begin{gathered}
p_{x}=\tau_{11, x}+\tau_{12, y}+\tau_{13, z} / \epsilon^{2}= \\
2 u_{x x}+4\left(W_{1}-W_{2}\right) u_{z}^{0} u_{z x} / \epsilon+\left(u_{y}+v_{x}\right)_{y}+2\left(W_{1}-W_{2}\right) u_{z}^{0} v_{z y} / \epsilon+u_{z z} / \epsilon^{2} .
\end{gathered}
$$


As $u_{x x}+u_{y y}=0, \quad u_{x x}+v_{x y}=0, \quad u_{z x}+v_{z y}=0$, it follows

$$
p_{x}=2\left(W_{1}-W_{2}\right) u_{z}^{0} u_{z x} / \epsilon+u_{z z} / \epsilon^{2} .
$$

We have obtained $p_{z}=0$ - see (35) - then the last above relation gives us

$$
p_{x z}=2\left(W_{1}-W_{2}\right)\left(u_{z}^{0} u_{z x}\right)_{z} / \epsilon+u_{z z z} / \epsilon^{2}=0 .
$$

Now we can justify the expression of $f$ used in (26), as follows.

Proposition 6. As before, we suppose $\exp (\sigma t)<1, \quad\left(W_{1}-W_{2}\right)=O(1)$. If the condition (43) holds and

$$
\beta=\epsilon^{2} / 27
$$

then $f(z)=\beta u^{0}(z)$ verifies (52) with the precision order $O(\epsilon)$.

Proof. We have $\max _{n}\{n \exp (-n \alpha)\}=1 /(\alpha e)$, then $\left|\left(u_{z}^{0} u_{z x}\right)_{z}\right| \leq 144 \beta /(\alpha e)$. It follows

$$
P X Z 1:=2\left(W_{1}-W_{2}\right)\left|\left(u_{z}^{0} u_{z x}\right)_{z}\right| \frac{1}{\epsilon} \leq 2\left(W_{1}-W_{2}\right) 144 \frac{\epsilon}{27} \cdot \frac{1}{11\left(W_{1}-W_{2}\right)}<\epsilon<<1 .
$$

As $u_{z z z}^{0}=0$, we conclude that $f(z)=\beta u^{0}(z)$ verifies (52) with the precision order $O(\epsilon)$. The condition (53) is in agreement with the hypothesis $\beta=O\left(\epsilon^{2}\right)$ used in (26).

\section{Conclusions}

A large part of the numerical results concerning the flow of Oldroyd-B fluids failed when the Weissenberg is near 1. In this paper we prove that this kind of instability is due to the model, and not to the numerical methods - at least in the case of Hele-Shaw displacements.

The basic solution (5) - (10) is considered, which governs the steady displacement of an Oldroyd-B fluid by air in a HeleShaw cell. We obtain the perturbations system (22) - (25) and get the growth constant corresponding to the particular perturbations (26). The dimensionless quantities are introduce in the relations (28) and we get the dimensionless (significant) constitutive relations (32), (33), (34), (39), (51), where the Weissenberg numbers $W_{i}$ are verifying $W_{i}=O(1), i=1,2$.

The perturbations (26) display bounded values for partial derivatives of $(u, v)$ in terms of $x, y, \forall x \geq 0$. The condition (43) imposed on the parameter $\alpha$ (appearing in the perturbations (26)) allows us to obtain the approximate expressions (38), (50), (48) for some components of the extra-stress tensor $\tau$ and for the growth constant $\sigma$.

If $\alpha=0$, then the perturbations (26) leads us to very high values of the derivatives of $(u, v)$ with respect to $x, y$ near the interface $x=0$. In this case, the estimates and the expressions given in Propositions 3 and 5 can not be obtained - see also Remark 2 .

In the case $W_{1}=W_{2}$, the growth rate (48) is quite similar with the Saffman-Taylor formula for a Newtonian fluid displaced by air in a Hele-Shaw cell. As we consider a 3D Hele-Shaw cell, the formula (48) contains the new term ( $-\gamma n)$ in the numerator and the new term $n^{2}\left(\epsilon^{2} / 6\right)$ in the denominator.

On the other hand, when $W_{1}=W_{2}$ (that means $\lambda_{1}=\lambda_{2}$ ) our basic flow is Newtonian. Indeed, consider the tensor $\mathbf{A}=\tau^{0}-\mu 2 \mathbf{S}^{0}=a_{i j}, \quad \mathbf{A}=\mathbf{A}^{T}$, then the constitutive relations (7) gives us

$$
\begin{gathered}
\tau^{0}-\lambda_{1}\left(\mathbf{V}^{0} \tau^{0}+\tau^{0} \mathbf{V}^{0 T}\right)=\mu\left\{\mathbf{2} \mathbf{S}^{0}-\lambda_{1}\left(\mathbf{V}^{0} \mathbf{2} \mathbf{S}^{0}+\mathbf{2} \mathbf{S}^{0} \mathbf{V}^{0 T}\right)\right\} \\
\mathbf{A}-\lambda_{1}\left(\mathbf{V}^{0} \mathbf{A}+\mathbf{A} \mathbf{V}^{0 T}\right)=0 .
\end{gathered}
$$

We have:

$$
\mathbf{V}^{0}=\left(\begin{array}{ccc}
0 & 0 & u_{z}^{0} \\
0 & 0 & 0 \\
0 & 0 & 0
\end{array}\right), \quad \mathbf{V}^{0} \mathbf{A}+\mathbf{A} \mathbf{V}^{0 T}=\left(\begin{array}{ccc}
2 u_{z}^{0} a_{31} & u_{z}^{0} a_{32} & u_{z}^{0} a_{33} \\
u_{z}^{0} a_{32} & 0 & 0 \\
u_{z}^{0} a_{33} & 0 & 0
\end{array}\right)
$$

and the relation (54) becomes

$$
\left(\begin{array}{ccc}
a_{11}-2 \lambda_{1} u_{z}^{0} a_{31} & a_{12}-\lambda_{1} u_{z}^{0} a_{32} & a_{13}-\lambda_{1} u_{z}^{0} a_{33} \\
a_{21}-\lambda_{1} u_{z}^{0} a_{32} & a_{22} & a_{23} \\
a_{31}-\lambda_{1} u_{z}^{0} a_{33} & a_{32} & a_{33}
\end{array}\right)=0 .
$$


First we get $a_{22}=a_{23}=a_{33}=0$. The third row gives $a_{31}=0$, from the second row it follows $a_{21}=0$ and the first row is giving $a_{11}=0$. Thus $\mathbf{A}=0$ and $\tau^{0}-\mu 2 \mathbf{S}^{0}=0$ - that means the basic flow (54) is Newtonian.

When $W_{1}-W_{2} \approx 0.408$, the growth constant formula (48) gives us a strong destabilization effect, compared with (Saffman \& Taylor, 1958) - see Remark 3 and Figure 2. Our dispersion curves are similar with the numerical results of (Wilson, 1990). We conclude that the instability for $W_{i}=O(1)$ is a property specific to the used flow pattern - in our case a Hele-Shaw displacement.

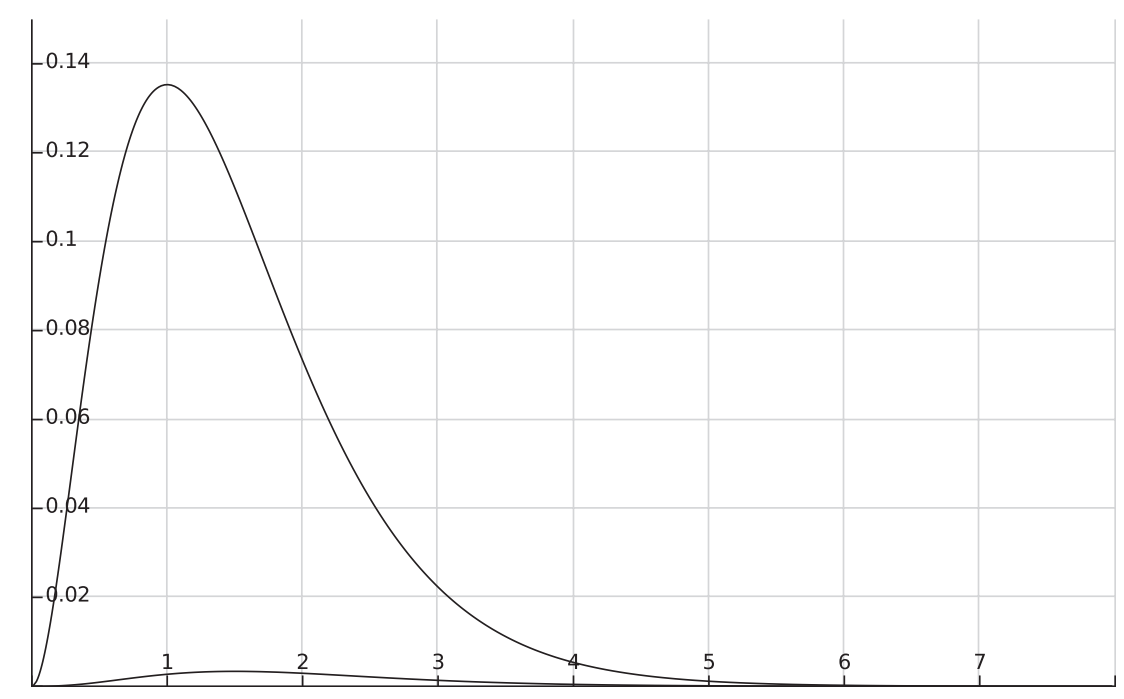

Figure 1. $\epsilon n^{3} \exp (-n \alpha)($ lower $)$ and $n^{2} \exp (-n \alpha)$ (upper) for $\alpha=2, \epsilon=0.02$.

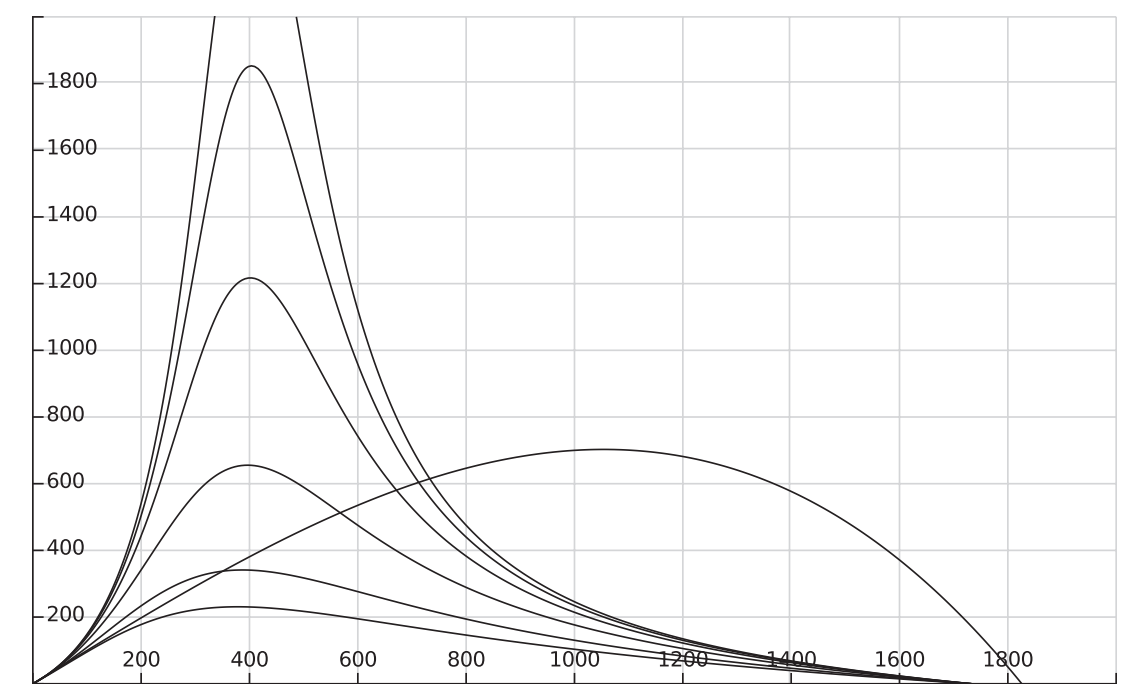

Figure 2. Plot of (48) compared with (49). $\epsilon=0.006, \gamma=0.1$, $W_{2}=0, W_{1}=0.1$ (lower), 0.2, 0.3, 0.35, 0.37, 0.38 (upper). 


\section{References}

Bear, J. (1972). Dynamics of Fluids in Porous Media, Courier Corporation.

Bird, R. B., \& Stewart, W. E. (1960). Transport phenomena, Vol. 1: Fluid Mechanics, John Wiley and Sons, N Y.

Fosdik, R. L., \& Rajakopal, K. D. (1979). Anomalous features in the model of "second order fluids", Arch. Rat. Mech. Anal., 70, 145-152. https://doi.org/10.100/BF00250351

Guillope, C., \& Saut, J. C. (1992). Mathematical problems arising in differential models for viscoelastic fluids, J.F. Rodrigues and A. Sequeira eds., Mathematical Topics in Fluid Mechanics, Longman, Halow, 64-92.

Hele-Shaw, H. S. (1898). The flow of water, Nature, 28, 34-36. https://doi.org/10.1038/058034a0

Lamb, Sir Horace (1933). Hydrodynamics, Cambridge University Press, Cambridge.

Miroshnichenko, D. S. (2001). Anisotropic second-order fluid, J. Mathematical Sciences, 103(2), 185-190(Engl.). https://doi.org/10.1023/A:1026694329838

Mora, S., \& Manna, M. (2009). Saffman-Taylor instability for generalized Newtonian fluids, Phys. Rev. E, 80, 016308. https://doi.org/10.1103/PhysRevE.80.016308

Mora, S., \& Manna, M. (2010). Saffman-Taylor instability of viscoelastic fluids: From viscous fingering to elastic fractures, Phys. Rev. E, 81, 026305. https://doi.org/10.1103/PhysRevE.81.026305

Nase,J., Lindner, A., \& Creton, C.(2008). Pattern formation during deformation of a confined visco elastic layer: From a viscous liquid to a soft elastic solid, Phys.Rev.Lett., 101, 074503. https://doi.org/10.1103/PhysRevLett.101.074503

Nittman, J., Daccord, G., \& Stanley, H. E. (1985). Fractal growth viscous fingers: quantitative characterization of a fluid instability phenomenon, Nature, 314(6007), 141-144, 141-4 CODEN: NATUAS; ISSN: 0028-0836; English.

Renardy, M. (2000). Mathematical analysis of viscoelastic flow, CBMS-NSF Regional Conference Series in Applied Mathematics, 73, Society for Industrial and Applied Mathematics (SIAM), Philadelphia, PA.

Saffman, P. G., \& Taylor, G. I. (195). The penetration of a fluid into a porous medium or Hele-Shaw cell containing a more viscous liquid, Proc. Roy. Soc. London, A, Math. Phys. Sci., 245, 312-329. https://doi.org/10.1098/rspa.1958.0085

Schowalter, W. R. (1978). Mechanics of Non-Newtonian Fluids, Pergamon Press, New York.

Truesdell, C., \& Noll, W. (1965). The non-linear filed theories of mechanics, Encyclopedia of Physics (ed. S. Fluggel) vol III/3, Springer Verlag.

Van Damme, H., Alsac, H. E., Laroche, C., \& Gatineau, L. (1988). On the respective roles of low surface tension and non-Newtonian rheological properties in fractal fingering, EPL (Europhysics Letters), 5(1), 25-38. https://doi.org/10.1209/0295-5075/5/1/005

Wilson, S. D. R. (1990). The Taylor-Saffman problem for a non-Newtonian liquid, Journal of Fluid Mechanics, 220, 413-425. https://doi.org/10.1017/S0022112090003329

Zhao, H., \& Maher, J. V. (1993). Associating-polymer effects in a Hele-Shaw experiment, Phys. Rev. E, 47, 4278-4283. https://doi.org/10.1103/PhysRevE.47.4278

\section{Copyrights}

Copyright for this article is retained by the author(s), with first publication rights granted to the journal.

This is an open-access article distributed under the terms and conditions of the Creative Commons Attribution license (http://creativecommons.org/licenses/by/4.0/). 